\title{
Evolution of Hydrodynamic Instability on Planar Jelly Interface Driven by Explosion*
}

\author{
Tao Wang, Jingsong Bai, Wenbin Huang, Yang Jiang, Liyong Zou, Ping Li, Duowang Tan \\ Institute of Fluid Physics, China Academy of Engineering Physics, Mianyang, China \\ Email: wtaoxp@21cn.com
}

Received April 26, 2012; revised May 20, 2012; accepted May 30, 2012

\begin{abstract}
A high precision numerical algorithm MVPPM (multi-viscous-fluid piecewise parabolic method) is proposed and applied to study the multi-viscous-fluid dynamics problems. Three planar jelly experiments with periodic cosine perturbation on the initial interface have been conducted and numerically simulated by MVPPM. Good agreement between experimental and numerical results has been achieved, including the shape of jelly interface, the displacements of front face of jelly layer, bubble top and spike head. The effects of initial conditions (including amplitude and wave length of perturbation, thickness of jelly layer, etc.) on the evolution of the jelly interface have been numerically analyzed. It is found that the key affecting factors are the perturbation amplitude and thickness of jelly layer. The hydrodynamic instability on double planar jelly layers driven by explosion has been investigated numerically to examine their laws of evolution, and an interesting phenomenon is observed.
\end{abstract}

Keywords: MVPPM; Jelly; Hydrodynamic Instability; Explosion

\section{Introduction}

The hydrodynamic interfacial instability is a very important physical phenomenon in a variety of man-made applications and natural phenomena such as inertial confinement fusion (ICF), high-speed combustion and astrophysics (i.e. supernova explosion). It can induce the turbulent mixing in the late times and has gained much attention for many years. According to the acting force on an interface between two different fluids, the hydrodynamic interfacial instability can be classified as the Richtmyer-Meshkov [1,2], Rayleigh-Taylor [3,4] and Kelvin-Helmholtz instability [5] (RMI, RTI and KHI), in which the interface experiences an impulsive acceleration (such as shock wave), a constant acceleration (gravity, for example) or a shear stress.

Various experiments [6-11] have been designed to study the interfacial instability. However, it is difficult to generate a well-defined and well-controlled, sharp initial interface between two fluids. One solution has been to initially separate two fluids using a thin membrane, then the membrane is broken into small pieces by the passing shock wave. However, these membrane fragments can influence the flow, and it is difficult to assess the effects of the broken fragments on the development of the interfacial instability. Other experiments have attempted to

*The authors thank supports from the National Natural Science Foundation of China (Grant No. 11072228, 11002129). avoid the effects of membranes by implementing systems in which the heavy gas is inserted into the test section from a container by a jet at the upper wall of shock tube, and flows out by an opposite center-to-center hole, the initial interface shaped with the jet is created [12-16]. However, because the diffusion coefficients of gases are large, this membraneless technique generates a thick diffusive interface. It will cause the uncertainty of initial conditions of interface, and they are often backward estimated by numerical simulations $[17,18]$. Another novel technique, which is developed by VNIIEF [19], is that the jelly interface is driven by the mixtures of $\mathrm{C}_{2} \mathrm{H}_{2}$ and $\mathrm{O}_{2}$ explosion. On one hand, the jelly has a sufficient strength allowing to fabricate the shells and layers of complicated form in no need of any membrane and to resist the deformation caused by gravity. On the other hand, it also has a good transparency to observe the hydrodynamic process of interface development easily. LLNL had done some studying works of jelly ring interface [20-22].

With the help of modern computers, numerical simulation has become a very useful and powerful means to study the interfacial instability. It allows us to examine the data in detail far beyond the capability of experiments. Many new numerical results have been reported [23-28]. In this paper, the hydrodynamic instability on planar jelly interface driven by explosion is studied by experiments and numerical simulations using the high precision nu- 
merical algorithm MVPPM, which is developed by combining the volume of fluid (VOF) methodology and piecewise parabolic method (PPM). Three planar jelly experiments with different periodic cosine perturbations on the initial interface have been conducted at the National Key Laboratory of Shock Wave and Detonation Physics (LSD). The effects of initial conditions (includeing amplitude and wave length of perturbation, thickness of jelly layer, etc.) on the evolution of the jelly interface have been analyzed numerically by MVPPM. Then the hydrodynamic instability on double planar jelly layers driven by explosion has been investigated numerically.

\section{Numerical Algorithm}

\subsection{Governing Equations}

When considering the viscosity and heat-conduction, the governing equations for multi-viscous-fluid can be written as follows in tensor notation:

$$
\left\{\begin{array}{l}
\frac{\partial \rho}{\partial t}+\frac{\partial \rho u_{j}}{\partial x_{j}}=0 \\
\frac{\partial \rho u_{i}}{\partial t}+\frac{\partial \rho u_{j} u_{i}}{\partial x_{j}}=-\frac{\partial p}{\partial x_{i}}+\frac{\partial \sigma_{i j}}{\partial x_{j}} \\
\frac{\partial \rho E}{\partial t}+\frac{\partial\left(\rho u_{j} E+p u_{j}\right)}{\partial x_{j}}=-\frac{\partial q_{j}}{\partial x_{j}}+\frac{\partial\left(u_{i} \sigma_{i j}\right)}{\partial x_{j}} \\
\frac{\partial Y^{(s)}}{\partial t}+u_{j} \frac{\partial Y^{(s)}}{\partial x_{j}}=0 \quad s=1,2, \cdots, N-1
\end{array}\right.
$$

Here, $i$ and $j$ represent the three directions of $x, y, z$ respectively; $\rho, u_{k}(k=i, j), p$ are the fluid density, velocity and pressure; $E$ is the total energy per unit mass; $N$ is the types of fluid, $Y^{(s)}$ is the volume fraction of the $s^{\text {th }}$ fluid and satisfies $\sum Y^{(s)}=1 ; \sigma_{i j}$ is the Newtonian fluid viscosity stress tensor:

$$
\sigma_{i j}=\mu\left(\frac{\partial u_{i}}{\partial x_{j}}+\frac{\partial u_{j}}{\partial x_{i}}-\frac{2}{3} \delta_{i j}\left(\frac{\partial u_{k}}{\partial x_{k}}\right)\right)
$$

where $\mu$ is the fluid viscosity; $q_{j}$ is the energy flux per unit time and space, $q_{j}=-\lambda \partial T / \partial x_{j}, \lambda$ is the efficient heat-conduction coefficient, $T$ is the temperature.

The equation of state is the "Stiffen Gas" form as:

$$
p=(\gamma-1) \rho e-\gamma \pi
$$

For gas, $\gamma$ is the ratio of specific heat, and $\pi=0$; for liquid and elastic closed-grained material described by impacting Hugoniot curve, $\gamma$ is the fitting constant of material, $\pi$ is constant with viscous stress tensor dimension; $e$ is the specific internal energy.

\subsection{Algorithm}

The physical process, as described by Equation (1), can be decomposed into three sub-processes, i.e., the calculations of inviscid flux, viscous flux and heat flux by operator splitting technique, and it is split up into two equations as follows:

$$
\left\{\begin{array}{l}
\frac{\partial \rho}{\partial t}+\frac{\partial \rho u_{j}}{\partial x_{j}}=0 \\
\frac{\partial \rho u_{i}}{\partial t}+\frac{\partial \rho u_{j} u_{i}}{\partial x_{j}}+\frac{\partial p}{\partial x_{i}}=0 \\
\frac{\partial \rho E}{\partial t}+\frac{\partial\left(\rho u_{j} E+p u_{j}\right)}{\partial x_{j}}=0 \\
\frac{\partial Y^{(s)}}{\partial t}+u_{j} \frac{\partial Y^{(s)}}{\partial x_{j}}=0 \quad s=1,2, \cdots, N-1
\end{array}\right.
$$

$$
\left\{\begin{array}{l}
\frac{\partial \rho}{\partial t}=0 \\
\frac{\partial \rho u_{i}}{\partial t}=\frac{\partial \sigma_{i j}}{\partial x_{j}} \\
\frac{\partial \rho E}{\partial t}=-\frac{\partial q_{j}}{\partial x_{j}}+\frac{\partial\left(u_{i} \sigma_{i j}\right)}{\partial x_{j}} \\
\frac{\partial Y^{(s)}}{\partial t}=0 \quad s=1,2, \cdots, N-1
\end{array}\right.
$$

The inviscid flux is calculated by PPM for multi-fluids to solve Equation (4), and the PPM of two-step Lagrange-Remapping algorithm in one time step can be divided into four steps: 1) the piecewise parabolic interpolation of physical quantities; 2) solving the Riemann problems approximately; 3) marching of the Lagrange equations; 4) remapping the physical quantities back to the stationary Euler meshes. Then the viscous flux and heat flux are calculated based on the computation of inviscid flux by using second-order spatial center difference and two-step Runge-Kutta time marching to solve Equation (5). The detailed description of numerical algorithm is referred in Ref. [29].

\section{Results and Discussions}

\subsection{Planar Jelly Experiment and Its Numerical Simulations}

The planar jelly experiment was carried out at LSD. The experimental equipment is illustrated in Figure 1, in which the inner cross section is $40 \mathrm{~mm} \times 40 \mathrm{~mm}$. The red part is the jelly layer. The cavity (driver section with length of $\mathrm{L}_{\mathrm{E}}=75 \mathrm{~mm}$ ) below jelly layer is filled with the mixture of $\mathrm{C}_{2} \mathrm{H}_{2}$ and $\mathrm{O}_{2}$ at one atm. The gaseous mixture detonates through discharge, and the instantaneous pressure of explosive products (EP) is measured to be $18 \mathrm{~atm}$, the Mach number of shock wave after explosion is about 


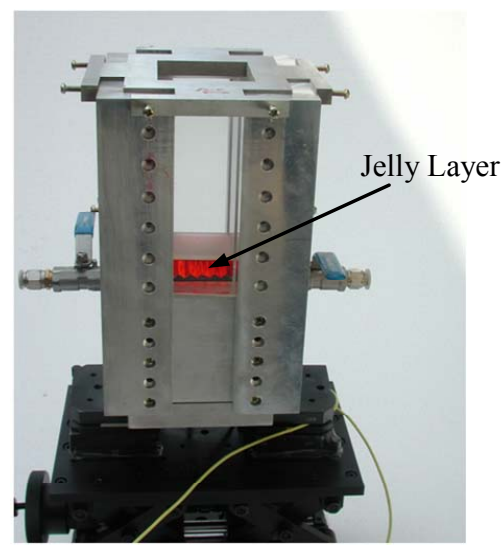

Figure 1. Experimental equipment.

3.592. One solid layer (plexiglass with thickness of $3 \mathrm{~mm}$ ) overlays the jelly layer, which keeps the planeness of front face of jelly layer when it is moving. The upper part of tube opens out to the atmosphere. The process of development of jelly layer is visualized by a high speed photography (Photron FASTCAM-APX RS). The Schematic of experimental data acquisition process is displayed in Figure 2, the xenon lamp is used as the illumination, the camera is located at opposite position of lamp, the computer and camera are connected with the ignition device by the synchronous machine, the experimental images are actually integrated ones. The full resolution of camera is $1024 \times 1024$ pixel arrays with 10 bits per pixel. The frame rate can vary in the range between $50 \mathrm{~Hz}$ and $250,000 \mathrm{~Hz}$, and it is set to be $30,000 \mathrm{fps}$ in our experiment. The exposure time of camera is $2 \mu \mathrm{s}$.

The jelly consists of gelatin water solution with the concentration $3.5 \%$. Therefore, its initial density is about $1000 \mathrm{~kg} / \mathrm{m}^{3}$, and the Atwood number is about 0.998 . The initial properties of jelly and explosive products are listed in Table 1. Three periodic initial cosine perturbations are set on the EP/jelly interface. The amplitudes and wave lengths are:

1) $\eta=0.5 \mathrm{~mm}, \lambda=8 \mathrm{~mm}$;

2) $\eta=1.0 \mathrm{~mm}, \lambda=8 \mathrm{~mm}$;

3) $\eta=1.0 \mathrm{~mm}, \lambda=5 \mathrm{~mm}$.

The schematic of computational model is shown in Figure 3. The grid resolution is $0.2 \mathrm{~mm}$.

Because the process of GEM explosion is very transitory, so it is neglected and completes momently in our simulations. The jelly layer is shocked by the gaseous mixture explosion, and the hydrodynamic interfacial instability happens at the EP/jelly interface. Figure 4 shows the time evolvement of jelly interface for the perturbation of $\eta=1.0 \mathrm{~mm}$ and $\lambda=8 \mathrm{~mm}$, the left and right columns are experimental and numerical results, respectively. It can be seen that the periodic initial cosine perturbation develops into structures shaped with bubble and spike regularly and gradually. Numerical results agree

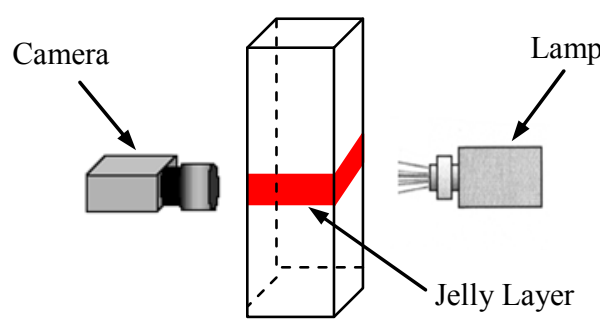

Figure 2. Schematic of experimental data acquisition process.

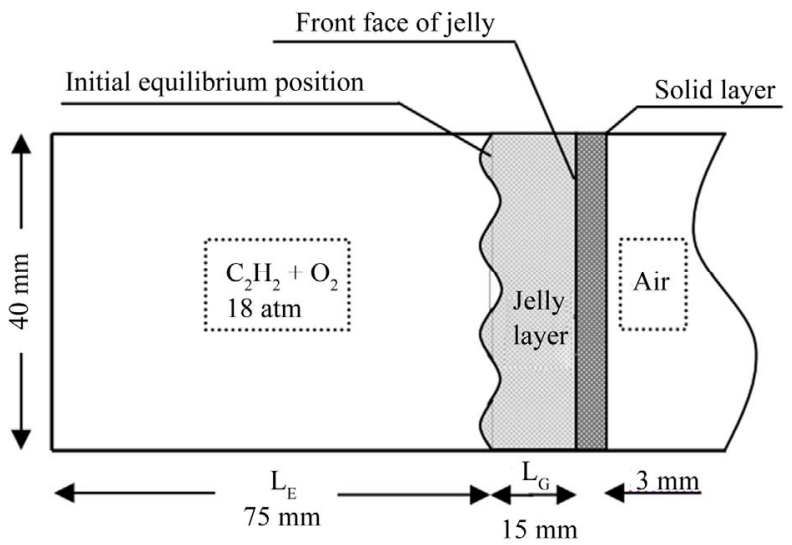

Figure 3. Schematic of computational model.

Table 1. Initial properties of jelly and explosive products.

\begin{tabular}{cccccc}
\hline Material & $\rho\left(\mathrm{kg} / \mathrm{m}^{3}\right)$ & $p(\mathrm{~atm})$ & $\mu(\mathrm{Pa} \cdot \mathrm{s})$ & $\gamma$ & $\pi(\mathrm{MPa})$ \\
\hline Jelly & 1000 & 1.0 & $1.002 \times 10 \mathrm{P}^{-3}$ & 7.0 & 300 \\
$\begin{array}{c}\text { Explosive } \\
\text { Products }\end{array}$ & 1.38 & 18.0 & $1.776 \times 10 \mathrm{P}^{-5}$ & 2.5 & 0.0 \\
\hline
\end{tabular}

well with the experimental ones qualitatively, including the shape of jelly interface, the positions of bubble top and spike head. Quantitative analysis between experiments and numerical simulations has been done by comparison of three geometric characteristic variables. These geometric characteristic variables are the displacements of front face of jelly layer $X_{1}$, bubble top $X_{2}$ and spike head $X_{3}$ relative to the initial equilibrium position of perturbation (white line), and is displayed in Figure 5. These experimental quantitative data of geometric characteristic variables are obtained by processing digitally the photographic images. Figure 6 shows the time histories of $X_{1}, X_{2}$ and $X_{3}$ for three kinds of initial perturbations. The comparison between numerical simulations and experiments reveals that good agreements are achieved except the displacement of spike head $\mathrm{X}_{3}$ at the late times. The errors may be resulted by the inaccurate measurements in experiments, because sometimes it is too difficult to determine the interface exactly, especially at the late times. In addition, the growth rates (slope of curve) of displacements $X_{1}, X_{2}$ and $X_{3}$ all increases with time gradually, and the bubble grows faster. 


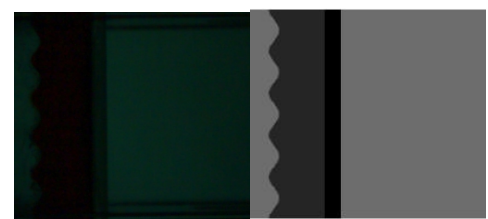

(a)

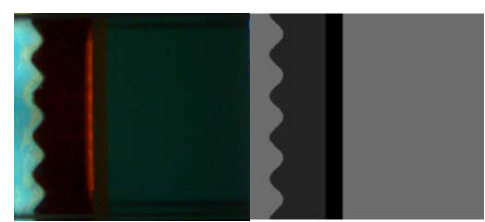

(b)

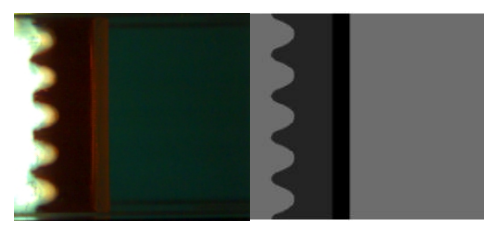

(c)

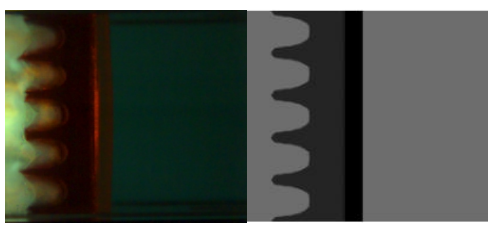

(d)

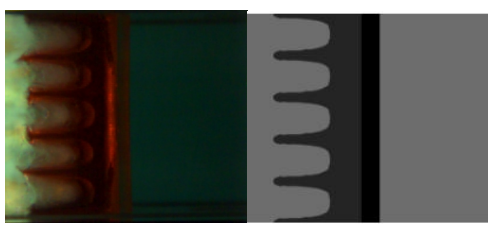

(e)

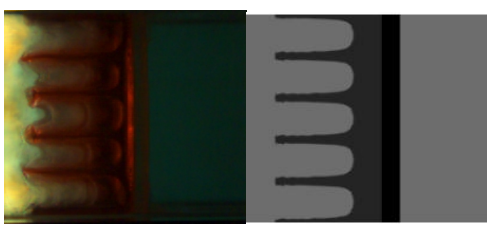

(f)

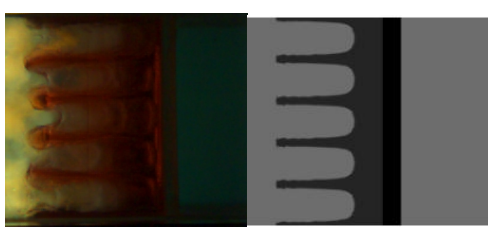

(g)

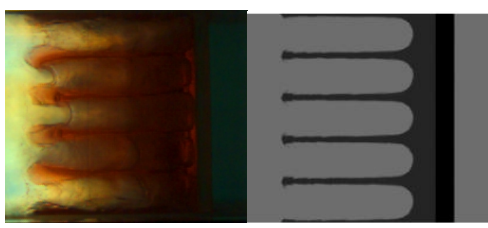

(h)

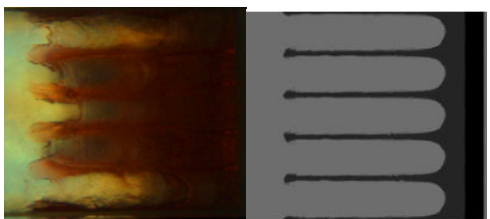

(i)

Figure 4. Time evolving of the jelly interface $(\eta=1.0 \mathrm{~mm}, \lambda$ $=8 \mathrm{~mm}$, left column: experiments, right column: simulations). (a) $t=0.0 \mathrm{~ms}$; (b) $t=0.1 \mathrm{~ms}$; (c) $t=0.2 \mathrm{~ms}$; (d) $t=0.3$ ms; (e) $t=0.4 \mathrm{~ms}$; (f) $t=0.5 \mathrm{~ms}$; (g) $t=0.6 \mathrm{~ms}$; (h) $t=0.7 \mathrm{~ms}$; (i) $t=0.8 \mathrm{~ms}$.

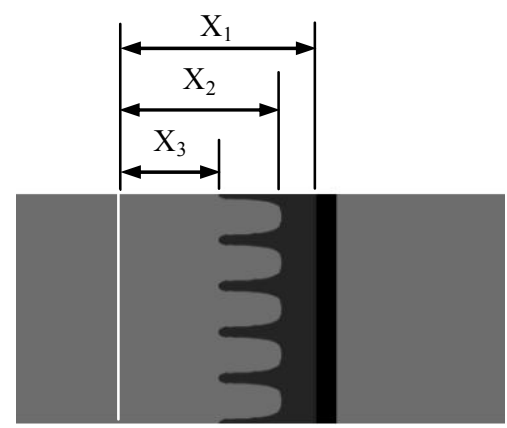

Figure 5. Schematic of displacements $X_{1}, X_{2}, X_{3}$.

\subsection{Numerical Analysis of the Effects of Initial Conditions on the Evolving of Jelly Interface}

In order to analyze the effects of initial conditions, including the amplitude and wave length of perturbation, thickness of jelly layer and width of explosion driver section, on the evolvement of jelly interface, sixteen models with different initial conditions have been calculated numerically by MVPPM based on the model in Sec. 3.1. The sixteen models have been divided into four groups according to the initial perturbation and listed in Table 2. Numerical images show that these jelly interfaces have a similar process of development. We define the width of mixing zone, $\mathrm{W}$, as the distance between bubble top and spike head, i.e. $\mathrm{W}=\mathrm{X}_{2}-\mathrm{X}_{3}$. Another quantity, $\mathrm{S}$, is the displacement of front face of jelly layer relative to its initial position. The effects of initial conditions on the evolution of the jelly interface are mainly analyzed by the comparisons of the width of mixing zone.

Figures 7(a)-(d) give the width of mixing zone vs. time for the models with same initial perturbation and different thickness of jelly layer and width of explosion driver section. Figures 7(e)-(h) give the width of mixing zone vs. time for the models with different initial perturbation and same thickness of jelly layer and width of explosion driver section. By the comparison, the following observations can be obtained. Firstly, the thickness of jelly layer has a major effect on the evolution of jelly interface, the width of mixing zone increases much faster 


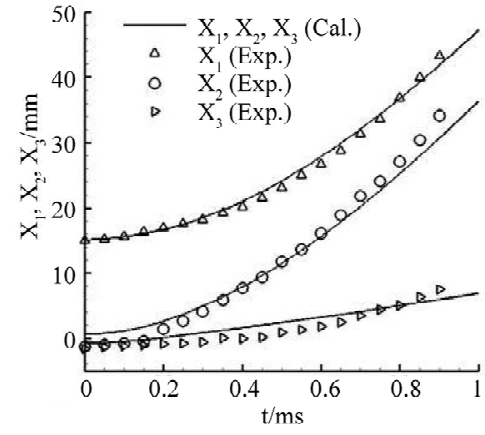

(a)

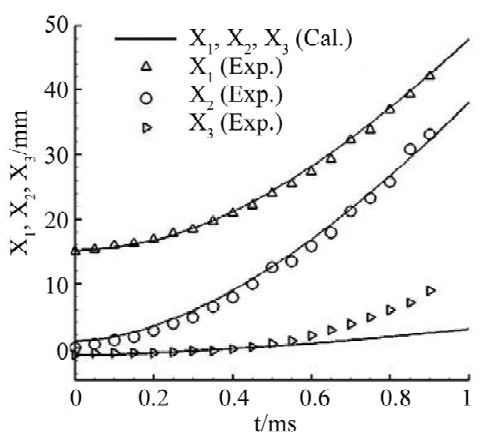

(b)

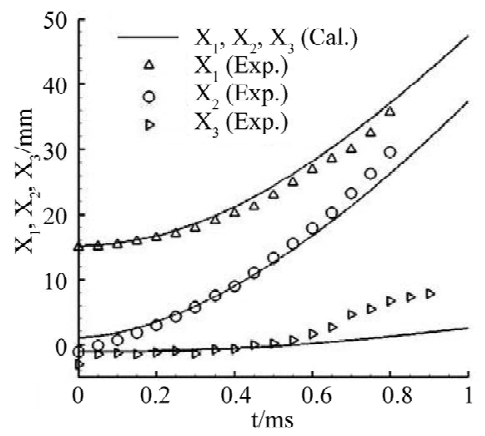

(c)

Figure 6. Time histories of $X_{1}, X_{2}$ and $X_{3}((a) \eta=0.5 \mathrm{~mm}, \lambda=8 \mathrm{~mm}$; (b) $\eta=1.0 \mathrm{~mm}, \lambda=8 \mathrm{~mm}$; (c) $\eta=1.0 \mathrm{~mm}, \lambda=5 \mathrm{~mm}$ ).

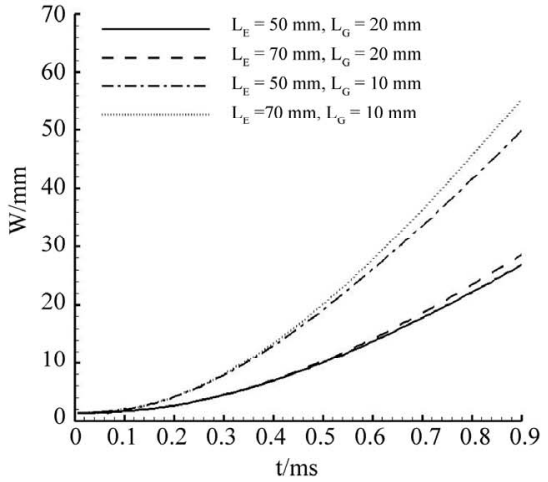

(a)

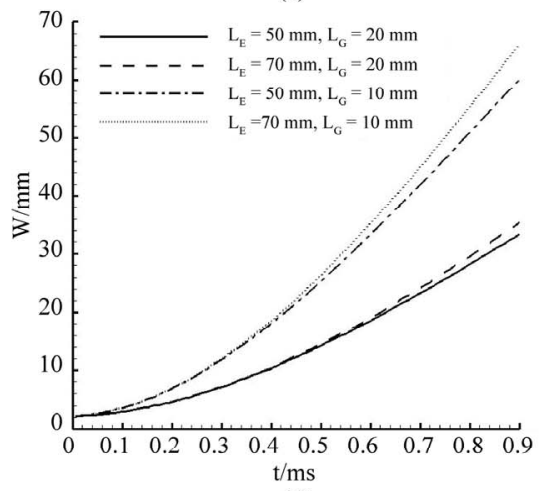

(d)

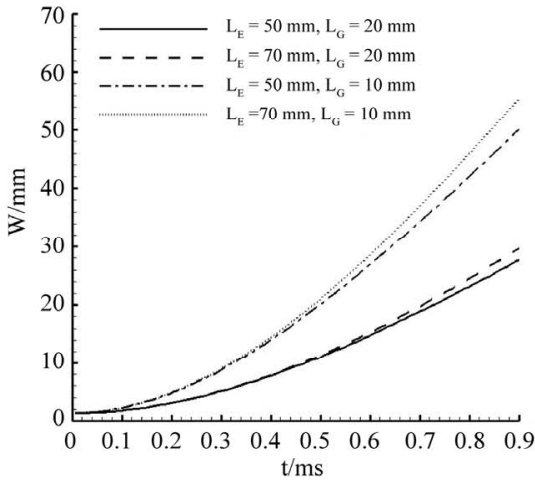

(b)

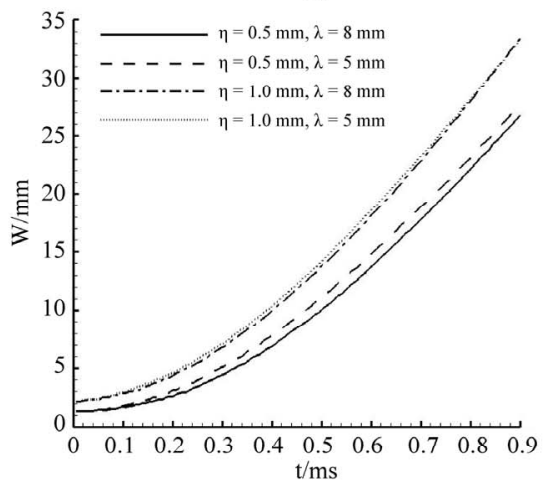

(e)

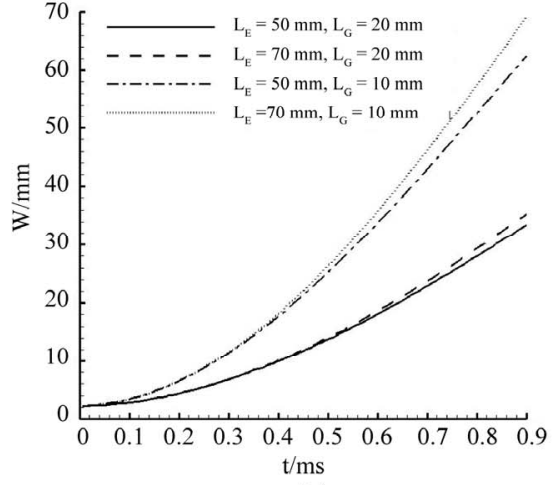

(c)

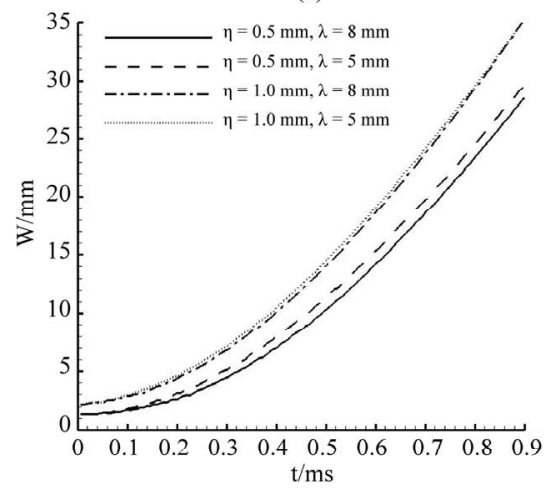

(f)

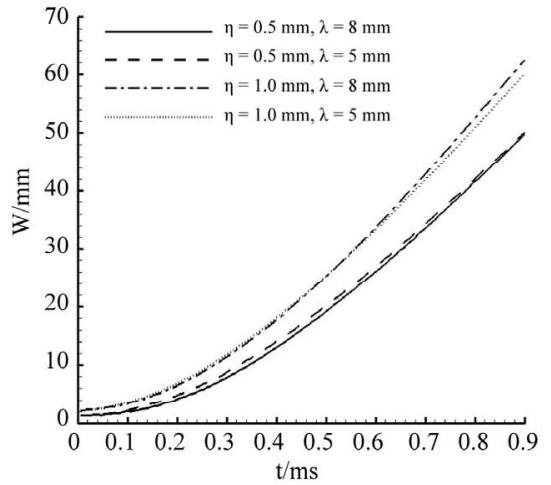

(g)

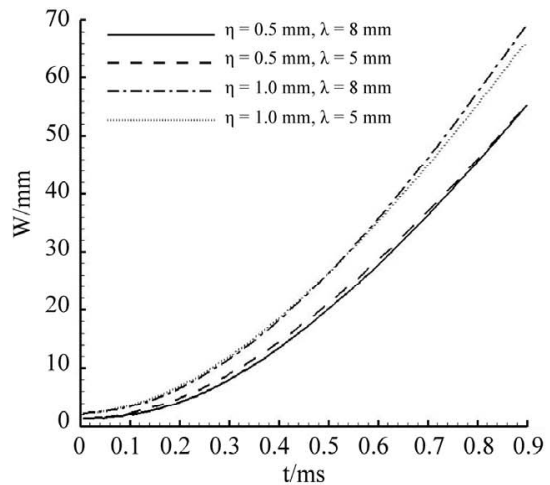

(h)

Figure 7. Width of mixing zone vs. time for different models. (a) $\eta=0.5 \mathrm{~mm}, \lambda=8 \mathrm{~mm}$; (b) $\eta=0.5 \mathrm{~mm}, \lambda=5 \mathrm{~mm}$; (c) $\eta=1.0$ $\mathrm{mm}, \lambda=8 \mathrm{~mm}$; (d) $\eta=1.0 \mathrm{~mm}, \lambda=5 \mathrm{~mm}$; (e) $\mathrm{L}_{\mathrm{E}}=50 \mathrm{~mm}, \mathrm{~L}_{\mathrm{G}}=20 \mathrm{~mm}$; (f) $\mathrm{L}_{\mathrm{E}}=70 \mathrm{~mm}, \mathrm{~L}_{\mathrm{G}}=20 \mathrm{~mm}$; (g) $\mathrm{L}_{\mathrm{E}}=50 \mathrm{~mm}, \mathrm{~L}_{\mathrm{G}}=$ $10 \mathrm{~mm}$; (h) $\mathrm{L}_{\mathrm{E}}=70 \mathrm{~mm}, \mathrm{~L}_{\mathrm{G}}=10 \mathrm{~mm}$. 
Table 2. Model parameters of single jelly layer.

\begin{tabular}{cccc}
\hline $\begin{array}{c}\text { Serial } \\
\text { Number }\end{array}$ & $\begin{array}{c}\mathrm{L}_{\mathrm{E}} \\
(\mathrm{mm})\end{array}$ & $\begin{array}{c}\mathrm{L}_{\mathrm{G}} \\
(\mathrm{mm})\end{array}$ & $\begin{array}{c}\text { Perturbation } \\
(\mathrm{mm})\end{array}$ \\
\hline $1-1$ & 50 & 20 & \\
$1-2$ & 70 & 20 & $y=0.5 \cos (2 \pi x / 8)$ \\
$1-3$ & 50 & 10 & \\
$1-4$ & 70 & 10 & \\
$2-1$ & 50 & 20 & \\
$2-2$ & 70 & 20 & \\
$2-3$ & 50 & 10 & \\
$2-4$ & 70 & 10 & \\
$3-1$ & 50 & 20 & \\
$3-2$ & 70 & 20 & \\
$3-3$ & 50 & 10 & \\
$3-4$ & 70 & 10 & \\
$4-1$ & 50 & 20 & \\
$4-2$ & 70 & 20 & \\
$4-3$ & 50 & 10 & \\
$4-4$ & 70 & 10 & \\
\hline
\end{tabular}

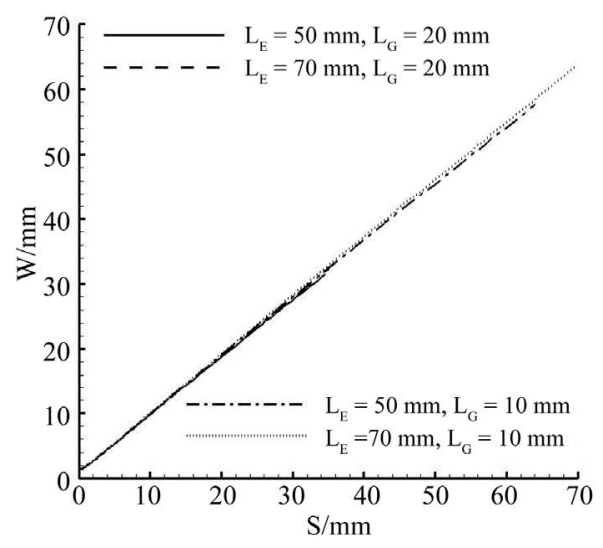

(a)

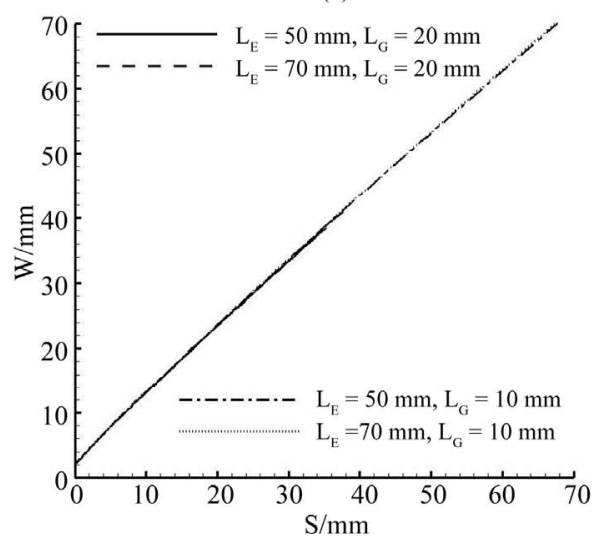

(c) when the thickness of jelly layer is smaller. Secondly, the width of explosion driver section has a relatively smaller effect on the evolution of jelly interface, especially when the thickness of jelly layer is larger. Thirdly, the amplitude of initial perturbation also has a major effect on the evolution of jelly interface, and the width of mixing zone grows faster when the amplitude is larger. Lastly, the wave length of initial perturbation has a less effect on the evolution of jelly interface. Figure 8 gives the width of mixing zone vs. the displacement of front face of jelly layer relative to its initial position for different models. It can be seen that the width of mixing zone changes linearly with the displacement of front face of jelly layer, i.e., the spatial growth rate of the width of mixing zone is a constant. Therefore, the key affecting factors on the evolution of jelly interface are the amplitude of initial perturbation and the thickness of jelly layer.

\subsection{Hydrodynamic Instability on Double Planar Jelly Layers}

In this section, the hydrodynamic instability on double planar jelly layer is numerically simulated. The objective is to study how the double planar jelly layers evolve

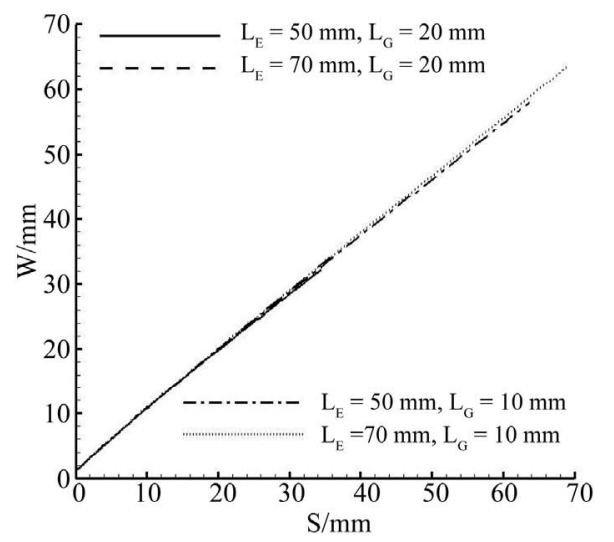

(b)

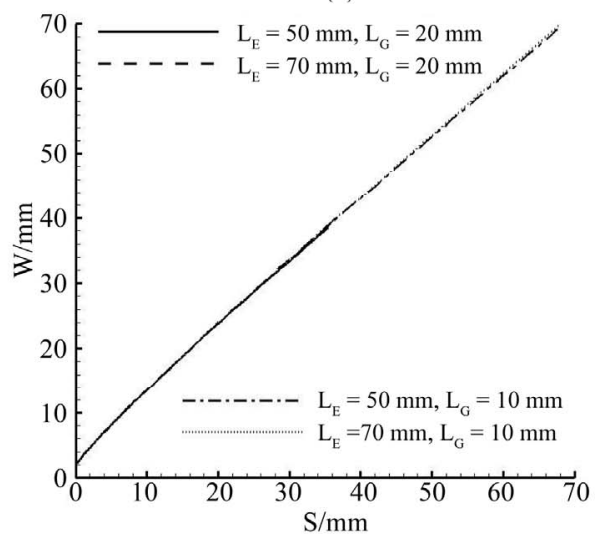

(d)

Figure 8. Width of mixing zone vs. the displacement of front face of jelly layer relative to its initial position for different models. (a) $\eta=0.5 \mathrm{~mm}, \lambda=8 \mathrm{~mm}$; (b) $\eta=0.5 \mathrm{~mm}, \lambda=5 \mathrm{~mm}$; (c) $\eta=1.0 \mathrm{~mm}, \lambda=8 \mathrm{~mm}$; (d) $\eta=1.0 \mathrm{~mm}, \lambda=5 \mathrm{~mm}$. 
driven by explosion. The schematic of computational model is shown in Figure 9. The thicknesses of jelly layer 1 and 2 are all $20 \mathrm{~mm}$, and denoted by LG1 and LG2 respectively. The jelly layer 1 has a periodic initial cosine perturbation on the EP/Jelly interface. The closed region between jelly layer 1 and 2 is filled with air at one atm. Eight models with different initial conditions have been calculated numerically and divided into two groups according as the jelly layer 2 has or has not periodic initial cosine perturbation. The model parameters are listed in Table 3.

Figures 10 and 11 give the numerical evolving images of double planar jelly layers when the jelly layer 2 has (1-1) and has not (2-1) periodic initial cosine perturbation respectively. It can be seen that the jelly layer 1 moves rightwards driven by gaseous mixture explosion, and the jelly interface 1 developed into the structures shaped with bubble and spike regularly and gradually. When the jelly layer 1 moves rightwards, the jelly layer 2 holds still, the jelly interface 2 has no any development, and the closed air is compressed. When the closed air is compressed to a degree, it starts to rebound. On the rebound, an interesting phenomenon happens on the jelly interface 1, which is that some new spikes are growing at the bubble top in the same direction (Figures 10(f)-(i) and Figures 11(f)-(i)). These new spikes grow much faster than their neighbors whose growing reduces obviously, and the original bubbles are split up into two ones symmetrically. The development of jelly interface 2 has a great difference on the rebound of closed air, whether it has initial perturbation. If jelly interface 2 has a periodic initial cosine perturbation, it will conglutinate with the jelly layer 1 at the perturbation crest, and the closed region is separated into several small regions, which are stretched gradually (Figures 10(e)-(i)); otherwise the jelly interface 2 has no any perturbation development, it can not conglutinate with the jelly layer 1 , and the jelly layer 2 moves rightwards driven by the compressed closed air as a whole (Figures 11(e)-(i)). The numerical simulations for other models show the same evolving laws.

Figure 12 shows the schematic of the mixing zone width of jelly interface 1 . Figure 13 shows the width of mixing zone of jelly interface 1 vs. time for different models. Several conclusions can be obtained. Firstly, under the driver of gaseous mixture explosion, the width of mixing zone of jelly interface 1 increases to an extremum gradually, so does its growth rate (slope of curve). At the intervals, the closed air is always compressed. After reaching the extremum, the width of mixing zone experiences a short-term negative growth, this is because the rebound of compressed closed air constrains its growth, then such effect of constraint weakens until disappearance, and the width of mixing zone increases again. Secondly, for the models with same initial perturbation on jelly interface 1 and 2 (It can be treated as the amplitude to be zero that jelly interface 2 has no initial

Table 3. Model parameters of double jelly layer.

\begin{tabular}{|c|c|c|c|c|c|c|}
\hline \multirow{2}{*}{ Serial Number } & \multirow{2}{*}{$\mathrm{L}_{\mathrm{E}}(\mathrm{mm})$} & \multirow{2}{*}{$\mathrm{L}_{\mathrm{G} 1}(\mathrm{~mm})$} & \multirow{2}{*}{$\mathrm{L}_{\mathrm{A}}(\mathrm{mm})$} & \multirow{2}{*}{$\mathrm{L}_{\mathrm{G} 2}(\mathrm{~mm})$} & \multicolumn{2}{|c|}{ Perturbation (mm) } \\
\hline & & & & & Jelly layer 1 & Jelly layer 2 \\
\hline $1-1$ & 50 & \multirow{8}{*}{20} & 20 & \multirow{8}{*}{20} & \multirow{4}{*}{$y=0.5 \cos (2 \pi x / 8)$} & \multirow{4}{*}{$y=0.5 \cos (2 \pi x / 5)$} \\
\hline $1-2$ & 70 & & 20 & & & \\
\hline $1-3$ & 50 & & 30 & & & \\
\hline $1-4$ & 70 & & 30 & & & \\
\hline $2-1$ & 50 & & 20 & & \multirow{4}{*}{$y=0.5 \cos (2 \pi x / 8)$} & \multirow{4}{*}{ None } \\
\hline $2-2$ & 70 & & 20 & & & \\
\hline $2-3$ & 50 & & 30 & & & \\
\hline $2-4$ & 70 & & 30 & & & \\
\hline
\end{tabular}

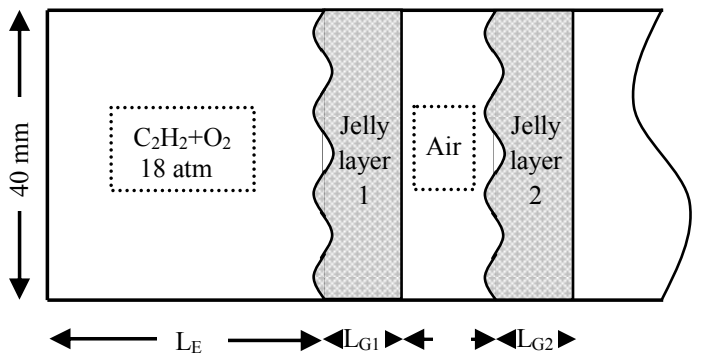

Figure 9. Schematic of computational model of double planar jelly layers. 


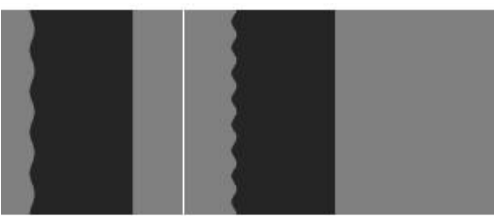

(a)

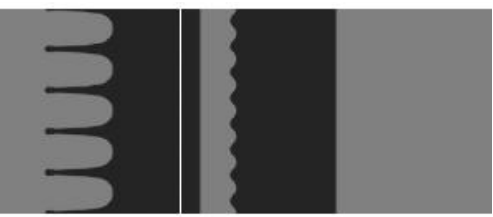

(d)

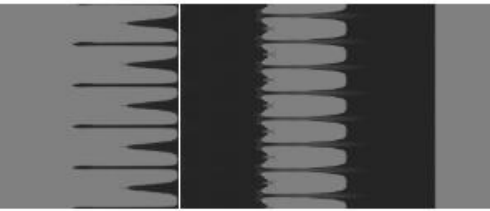

(g)

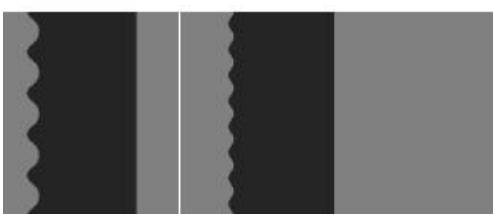

(b)

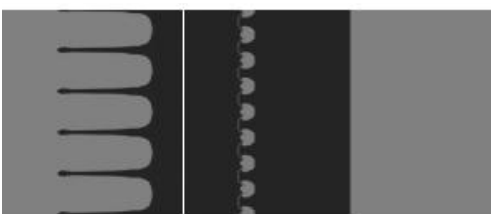

(e)

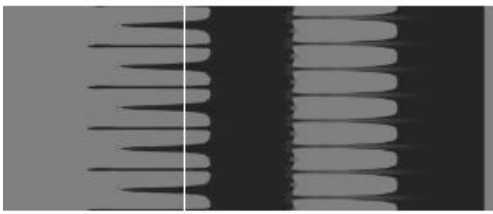

(h)

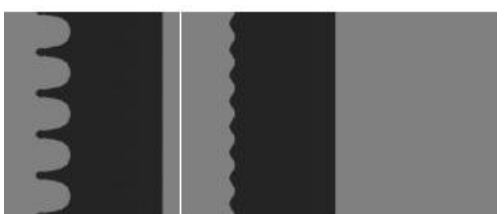

(c)

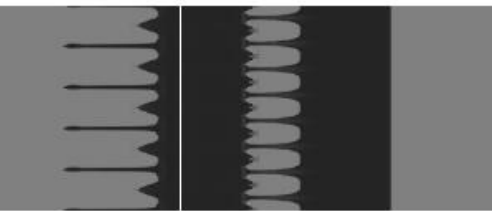

(f)

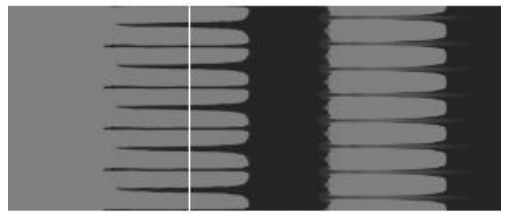

(i)

Figure 10. Numerical time evolving images of double planar jelly layers (1-1). (a) $t=0.0 \mathrm{~ms}$; (b) $t=0.2 \mathrm{~ms}$; (c) $t=0.4 \mathrm{~ms}$; (d) $t$ $=0.6 \mathrm{~ms}$; (e) $t=0.8 \mathrm{~ms}$; (f) $t=1.0 \mathrm{~ms}$; (g) $t=1.2 \mathrm{~ms}$; (h) $t=1.4 \mathrm{~ms}$; (i) $t=1.6 \mathrm{~ms}$.

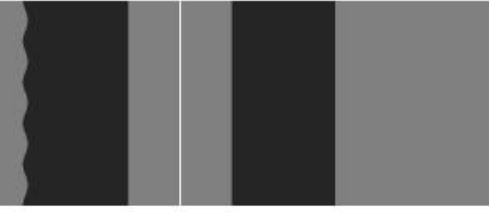

(a)

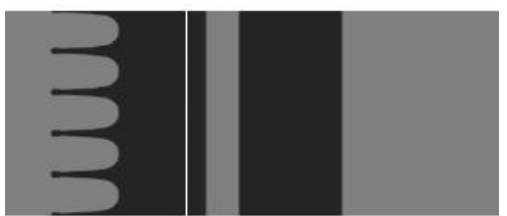

(d)

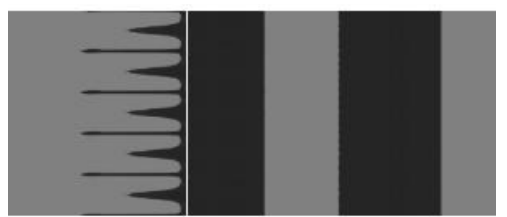

(g)

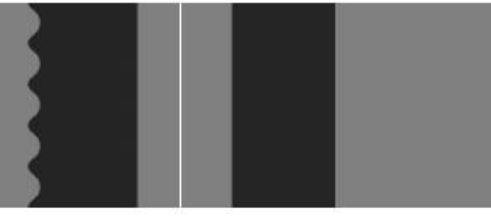

(b)

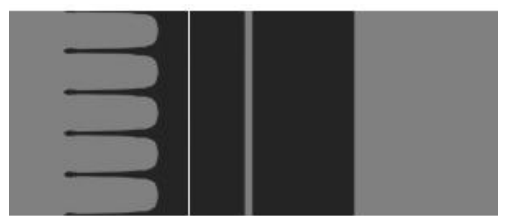

(e)

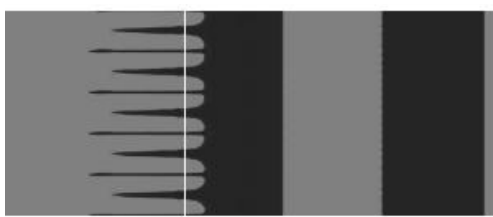

(h)

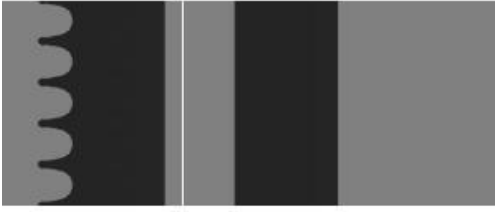

(c)

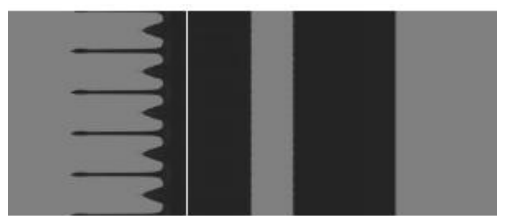

(f)

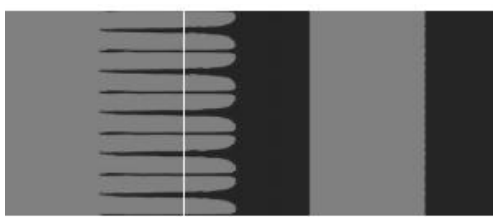

(i)

Figure 11. Numerical time evolving images of double planar jelly layers (2-1). (a) $t=0.0 \mathrm{~ms}$; (b) $t=0.2 \mathrm{~ms}$; (c) $t=0.4 \mathrm{~ms}$; (d) $t$ $=0.6 \mathrm{~ms}$; (e) $t=0.8 \mathrm{~ms}$; (f) $t=1.0 \mathrm{~ms}$; (g) $t=1.2 \mathrm{~ms}$; (h) $t=1.4 \mathrm{~ms}$; (i) $t=1.6 \mathrm{~ms}$.

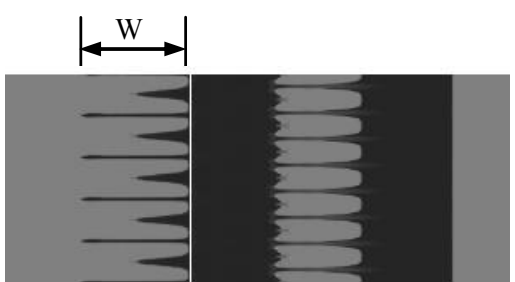

Figure. 12. Schematic of the mixing zone width of jelly interface 1. perturbation.), the width of explosion driver section almost can not affect the evolution of mixing zone before the rebound of compressed closed air. But on its rebound, the effect of width of explosion driver section enhances gradually, the width of mixing zone of jelly interface 1 increases faster for models with the larger width of explosion driver section. The width of closed air almost can not influence the growth of mixing zone too before its rebound. When the width of closed air is larger, it will 


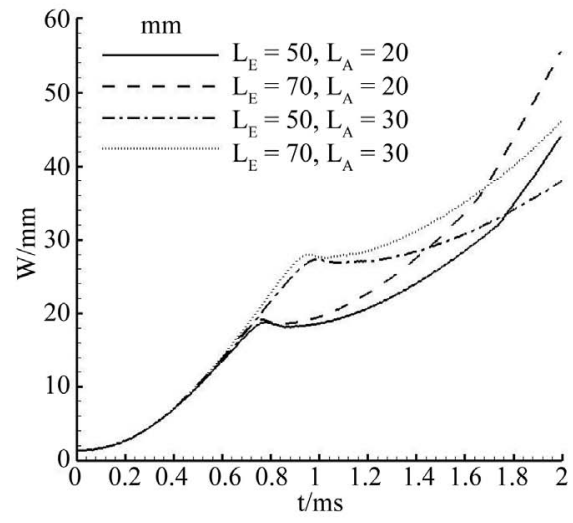

(a)

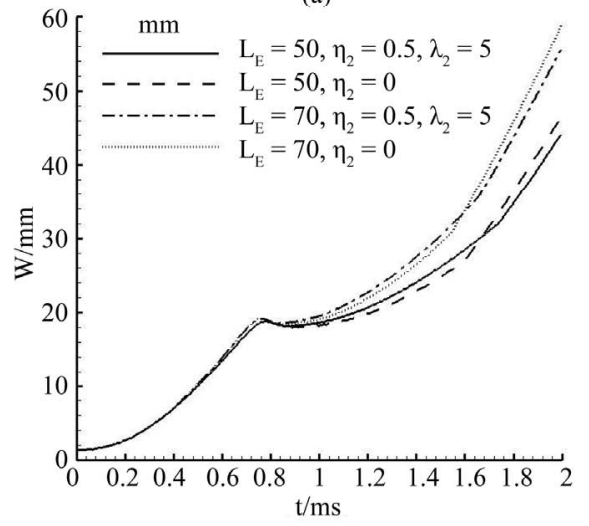

(c)

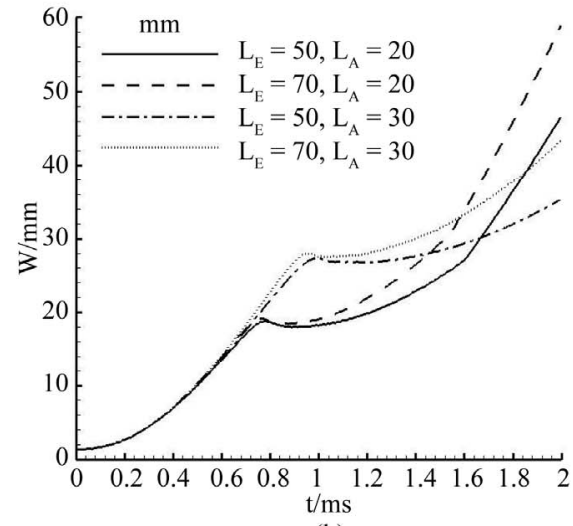

(b)

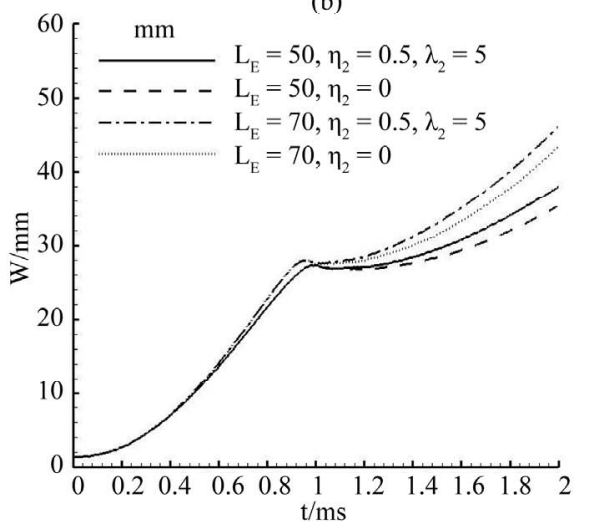

(d)

Figure 13. Width of mixing zone of jelly interface 1 vs. time for different models. (a) $y_{1}=0.5 \cos (2 \pi x / 8) \& y_{2}=0.5 \cos (2 \pi x / 5)$; (b) $y_{1}=0.5 \cos (2 \pi x / 8) \& y_{2}=0$; (c) $L_{A}=20 \mathrm{~mm}$; (d) $L_{A}=30 \mathrm{~mm}$.

pass through a long process of compression, and its rebound will lag behind. The width of mixing zone has a greater difference on the rebound of compressed closed air with a different size (Figures 13(a) and (b)). Thirdly, for the models with same width of explosion driver sec tion and closed air, the growth of mixing zone of jelly interface 1 almost can not be influenced before the rebound of compressed closed air whether the jelly interface 2 has initial perturbation. On the rebound, when the closed region is greater, the growth of mixing zone of jelly interface 1 has a larger difference whether the jelly interface 2 has initial perturbation (Figures 13(c) and (d)). Therefore, for the hydrodynamic instability on double planar jelly layers driven by explosion, the key affecting factors are the width of explosion driver section and closed air, and the initial perturbation on the jelly interface 2 .

\section{Conclusion}

In this paper, the hydrodynamic instability on planar jelly interface driven by explosion is studied by experiments and numerical simulations. Three single layer of planar jelly experiments with periodic initial cosine perturbation conducted at LSD are numerically simulated by high precision numerical algorithm MVPPM. There is good agreement between experiments and numerics. Then several single jelly layer of models are numerically simulated to analyze the effects of initial conditions (includeing amplitude and wave length of perturbation, thickness of jelly layer and width of explosion driver section) on the evolution of jelly interface. The key affecting factors are the perturbation amplitude and thickness of the jelly layer. The hydrodynamic instability on double planar jelly layers driven by explosion has been investigated numerically, in which the evolving law is greatly different from the one of single jelly layer, and the affecting factors are more.

\section{REFERENCES}

[1] R. D. Richtmyer, "Taylor Instability in Shock Acceleration of Compressible Fluids," Communications on Pure and Applied Mathematics, Vol. 13, No. 2, 1960, pp. 297 319. doi:10.1002/cpa.3160130207

[2] E. E. Meshkov, "Instability of the Interface of Two Gases Accelerated by a Shock Wave," Soviet Fluid Dynamics, Vol. 4, No. 5, 1969, pp. 101-104. doi:10.1007/BF01015969

[3] G. I. Taylor, "The Instability of Liquid Surfaces When 
Accelerated in a Direction Perpendicular to Their Planes. I," Proceedings of the Royal Society A, Vol. 201, No. 1065, 1950, pp. 192-196. doi:10.1098/rspa.1950.0052

[4] D. H. Sharp, "An Overview of Rayleigh-Taylor Instability," Physica D, Vol. 12, No. 1-3, 1984, pp. 3-18. doi:10.1016/0167-2789(84)90510-4

[5] S. Chandrasekhar, "Hydrodynamic and Hydromagnetic Stability," Oxford University Press, London, 1961.

[6] G. Dimonte and M. B. Schneider, "Turbulent RayleighTaylor Instability Experiments with Variable Acceleration," Physical Review E, Vol. 54, No. 4. 1996, pp. 37403743. doi:10.1103/PhysRevE.54.3740

[7] Y. A. Kucherenko1, A. P. Pylaev, V. D. Murzakov, et al., "Experimental Study into the Asymptotic Stage of the Separation of the Turbulized Mixtures in Gravitationally Stable Mode," In: R. Young, J. Glimm and B. Boston, Eds., Proceedings of the 5th International Workshop on Compressible Turbulent Mixing, Stony Brook University Press, New York, 1996.

[8] J. W. Jacobs and J. M. Sheeley, "Experimental Study of Incompressible Richtmyer-Meshkov Instability," Physics of Fluids, Vol. 8, No. 2, 1996, pp. 405-415. doi:10.1063/1.868794

[9] E. E. Meshkov, N. V. Nevmerzhitsky, V. A. Pavlovskii, et al., "Jelly Technique Applications in Evolution Study of Hydrodynamic Instabilities on Unstable Plane and Cylindrical Surfaces," In: R. Young, J. Glimm and B. Boston, Eds., Proceedings of the 5th International Workshop on Compressible Turbulent Mixing, Stony Brook University Press, New York, 1996.

[10] L. Houas, G. Jourdan, L. Schwaederlé, et al., “A New Large Cross-Section Shock Tube for Studies of Turbulent Mixing Induced by Interfacial Hydrodynamic Instability," Shock Waves, Vol. 13, No. 5, 2003, pp. 431-434. doi:10.1007/s00193-002-0173-y

[11] S. H. R. Hosseini and K. Takayama, "Experimental Study of Richtmyer-Meshkov Instability Induced by Cylindrical Shock Waves," Physics of Fluids, Vol. 17, No. 8, 2005, p. 084101. doi:10.1063/1.1964916

[12] P. M. Rightley, P. Vorobieff, R. Martin, et al., "Experimental Observations of the Mixing Transition in a ShockAccelerated Gas Curtain," Physics of Fluids, Vol. 11, No. 1, 1999, pp. 186-200. doi:10.1063/1.869911

[13] B. J. Balakumar, G. C. Orlicz, C. D. Tomkins, et al., "Simultaneous Particle-Image Velocimetry-Planar Laser-Induced Fluorescence Measurements of Richtmyer-Meshkov instability Growth in a Gas Curtain with and without Reshock," Physics of Fluids, Vol. 20, No. 12, 2008, p. 124103. doi:10.1063/1.3041705

[14] J. W. Jacobs, "The Dynamics of Shock Accelerated Light and Heavy Gas Cylinders," Physics of Fluids, Vol. 5, No. 9, 1993, pp. 2239-2247. doi:10.1063/1.858562

[15] C. D. Tomkins, K. P. Prestridge, P. M. Rightley, et al., "A Quantitative Study of the Interaction of Two RichtmyerMeshkov Unstable Gas Cylinders," Physics of Fluids, Vol. 15, No. 4, 2003, pp. 986-1004. doi:10.1063/1.1555802

[16] S. Kumar, P. Vorobieff, G. Orlicz, et al., "Complex Flow Morphologies in shock-Accelerated Gaseous Flows," Physica D, Vol. 235, No. 1-2, 2007, pp. 21-28.

\section{doi:10.1016/i.physd.2007.04.023}

[17] S. Gupta, S. Zhang and N. J. Zabusky, "Shock Interaction with a Heavy Gas Cylinder: Emergence of Vortex Bilayers and Vortex- Accelerated Baroclinic Circulation Generation," Laser and Particle Beams, Vol. 24, No. 3, 2003, pp. 443-448.

[18] S. Zhang, N. J. Zabusky, G. Z. Peng, et al., "Shock gases Cylinder Interactions: Dynamically Validated Initial Conditions Provide Excellent Agreement between Experiments and Numerical Simulations to Late-Intermediate Time," Physics of Fluids, Vol. 16, No. 5, 2004, pp. 1203-1216. doi:10.1063/1.1651483

[19] S. Andronov and E. E. Meshkov, "Computational and Experimental Studies of Hydro-Dynamic Instabilities and Turbulent Mixing," Los Alamos National Laboratory, Los Alamos, 1995

[20] B. Goodwin an S. Weir, "Rayleigh-Taylor Instability Experiments in a Cylindrically Convergent Geometry," Los Alamos National Laboratory, Los Alamos, 1996.

[21] K. O. Mikaelian, "Rayleigh-Taylor and Richtmyer-Meshkov Instabilities and Mixing in Stratified Spherical Shells," Physical Review A, Vol. 42, No. 6, 1990, pp. 3400-3420. doi:10.1103/PhysRevA.42.3400

[22] K. O. Mikaelian, "Rayleigh-Taylor and Richtmyer-Meshkov Instabilities and Mixing in Stratified Cylindrical Shells," Physics of Fluids, Vol. 17, No. 9, 2005, pp. 094105. doi:10.1063/1.2046712

[23] N. C. Hearn, T. Plewa, R. P. Drake, et al., "Flash Code Simulation of Rayleigh-Taylor and Richtmyer-Meshkov Instabilitites in Laser-Driven Experiments," Astrophysics and Space Science, Vol. 307, No. 1-3, 2007, pp. 227-231. doi:10.1007/s10509-006-9226-5

[24] H. Lee, H. Jin, Y. Yu, et al., "On Validation of Turbulent Mixing Simulations for Rayleigh-Taylor Instability," Physics of Fluids, Vol. 20, No. 1, 2008, p. 012102. doi:10.1063/1.2832775

[25] A. Marocchino, S. Atzeni and A. Schiavi, "Numerical Study of the Ablative Richtmyer-Meshkov Instability of Laser-Irradiated Deuterium and Deuterium-Tritium Targets," Physics of Plasmas, Vol. 17, No. 11, 2010, p. 112703. doi:10.1063/1.3505112

[26] L. Biferale, F. Mantovani and M. Sbragaglia, "High Resolution Numerical Study of Rayleigh-Taylor Turbulence Using a Thermal Lattice Boltzmann Scheme," Physics of Fluids, Vol. 22, No. 11, 2010, p. 115112. doi:10.1063/1.3517295

[27] L. F. Wang, W. H. Ye, W. S. Don, et al., "Formation of Large-Scale Structures in Ablative Kelvin-Helmholtz Instability," Physics of Plasmas, Vol. 17, No. 12, 2010, p. 122308. doi:10.1063/1.3524550

[28] M. A. Ullah, W. B. Gao and D. K. Mao, "Numerical Simulations of Richtmyer-Meshkov Instabilities Using Conservative Front-Tracking Method," Applied Mathematics and Mechanics, Vol. 32, No. 1, 2011, pp. 119-132. doi:10.1007/s10483-011-1399-x

[29] T. Wang, J. S. Bai, P. Li, et al., "The Numerical Study of Shock-Induced Hydrodynamic Instability and Mixing," Chinese Physics B, Vol. 18, No. 3, 2009, pp. 1127-1135. doi: $10.1088 / 1674-1056 / 18 / 3 / 048$ 